\title{
Facebook Support Groups for Rare Pediatric Diseases: Quantitative Analysis
}

\author{
Sarah Catrin Titgemeyer ${ }^{1}$; Christian Patrick Schaaf ${ }^{2,3}, \mathrm{MD}, \mathrm{PhD}$ \\ ${ }^{1}$ University of Cologne, Cologne, Germany \\ ${ }^{2}$ Institute of Human Genetics, Heidelberg University, Heidelberg, Germany \\ ${ }^{3}$ Institute of Human Genetics, University Hospital Cologne, Cologne, Germany
}

\section{Corresponding Author:}

Christian Patrick Schaaf, MD, PhD

Institute of Human Genetics

Heidelberg University

Im Neuenheimer Feld 366

Heidelberg, 69120

Germany

Phone: 496221565151

Email: christian.schaaf@med.uni-heidelberg.de

\begin{abstract}
Background: Loneliness, social isolation, and feeling disconnected from society are commonly experienced by parents of children with rare diseases and are, among others, important reasons for special supportive care needs. Social networking platforms are increasingly used for health communication, information exchange, and support. In the field of rare pediatric diseases, qualitative studies have shown that Facebook online support groups are utilized by and beneficial for persons affected by rare pediatric diseases. Nonetheless, the extent of this usage has not been investigated.

Objective: This study aims to provide a comprehensive quantitative analysis of the extent of Facebook usage as a tool for rare pediatric disease support groups and to explore factors that influence a disease's representation on Facebook. These results potentially offer important insights for future public health initiatives and give direction to further research that can give much needed support to parents of children with rare diseases.
\end{abstract}

Methods: We determined rare pediatric diseases using the inventory of the online portal Orphanet. Facebook support groups were identified by searching 5 synonymous disease descriptions using the group category search bar. Disease- and group-describing parameters were statistically analyzed using standard descriptive statistical methods.

Results: 6398 Facebook support groups, representing 826 diseases (19.5\% of all searched diseases), were found. $69 \%$ are private groups. Group type, size, activity (sum of posts, comments, and reactions calculated by Facebook), new memberships, and language varied largely between groups (member count: minimum 1, maximum 23,414; activity last 30 days: minimum 0, maximum 3606). The highest percentage of awareness and information groups was found for teratogenic diseases (18/68, 26\%). The odds of finding a Facebook group increased according to the level of information available about the disease: known prevalence (odds ratio [OR] 3.98, 95\% CI 3.39-4.66, $P<.001$ ), known disease type (OR 3.15, 95\% CI 2.70-3.68, $P<.001$ ), and known inheritance mode (OR 2.06, 95\% CI 1.68-2.52, $P<.001$ ) were all associated with higher odds of finding a Facebook group, as was dominant compared to nondominant inheritance (OR 2.05, 95\% CI 1.74-3.42, $P<.001$ ). The number of groups per disease increased with higher prevalence.

Conclusions: Facebook is widely used as a tool for support groups for rare pediatric diseases and continues to be relevant. Two-thirds of the groups are private groups, indicating group participants' need for privacy, which should be further explored. The advantages and limitations of Facebook as a tool for support groups in the field of rare diseases should be further investigated as it will allow health professionals to use Facebook more meaningfully in their counseling and guidance of affected individuals and their family members.

(JMIR Pediatr Parent 2020;3(2):e21694) doi: 10.2196/21694 


\section{KEYWORDS}

pediatric rare diseases; rare diseases; support group; online support; Facebook support group; social media; parent support; support group privacy; counseling

\section{Introduction}

\section{Background}

Many parents of children affected by rare diseases described caring for a child with a rare disease to be highly isolating — with loneliness, social isolation and feeling disconnected from society being mentioned as common problems [1]. Most parents had never come into contact with other parents of a child with a similar condition to that of their own child, and many were dissatisfied with the overall support that they had received for their child with a rare disease from any source [1]. Since rare diseases have per definition a very low prevalence (European definition of rare diseases: <1 per 2000) [2], affected individuals are often geographically dispersed. For many rare diseases there is a deficit of medical and scientific knowledge [2]. Rare diseases are often serious, chronic, and progressive, and persons affected by rare diseases are more psychologically, socially, economically, and culturally vulnerable [2]. Hence, parents of children with rare diseases have special supportive care needs [1].

Parent-to-parent peer support has been shown to have beneficial effects on parents of children with disabilities and children with additional needs [3]. Parents benefit from support groups most importantly by building social connections, gaining a sense of belonging [4,5], and developing a sense of control [5]. Support groups provide an environment for parent-to-parent support, which offers several benefits through improved social support. Participating parents can experience improved social connections [4], a heightened sense of control [5], higher family life congruence [6], and lower consequences of perceived stress [7].

Social networking platforms are increasingly used for health communication, information exchange, and support. Benefits of using social media for health-related online communication and community include connectedness, increased community support, and online support groups [8]. Several advantages of social media for online support have been identified, including international scope, unlimited number of participants, cost-effectiveness [9], and 24-hour availability [10]. However, there are limitations, such as questionable reliability [11], accuracy [9], quality [12], application to personal situations [11], and the possible misinterpretation [9] of information found online and on social media.

Founded in 2004, Facebook is one of the longest existing social networking platforms [13]. In the second quarter of 2019, Facebook reported 2.41 billion monthly active users [14]. Thus, chances are presumably high that another person affected by the same disorder also uses Facebook and would be eligible to form a support group. Facebook allows persons to connect independent of geographic location and offers options for both individual and group communication [13].

\section{Prior Work}

Parents of children with rare diseases are active internet users, search for information online [15], and use social media such as Facebook to communicate and link with others [12], showing that most parents are already familiar with Facebook and are, therefore, likely have the required social networking skills. Therefore, they could benefit by extending their Facebook usage to participation in support groups quite effortlessly.

To our knowledge, only little research exploring the specific topic of online support groups for rare pediatric diseases has been conducted so far. Content analyses of specific online and Facebook support groups have been performed (eg, on groups for cleft lip and palate, clubfoot, Hirschsprung disease, autism spectrum disorders, Dravet syndrome, and related epilepsy disorders [16-21]). Group members benefit from giving and receiving informational and emotional support and from connecting with others since meeting others with similar experiences has been shown to decrease isolation [10,16-19].

\section{Research Rationale}

Social support can provide several benefits for parents caring for children with chronic diseases, disabilities, additional needs, behavioral problems, and rare diseases [3-10,12,13,15,16,18,20]. Qualitative studies have shown that Facebook is utilized by and beneficial for persons affected by rare pediatric diseases. However, since these studies focused on specific conditions or groups, they failed to reflect the extent of support group usage and the overall representation of rare pediatric diseases on Facebook.

Our study therefore aimed to provide a comprehensive quantitative analysis of the extent of Facebook usage as a tool for rare pediatric disease support groups and at analyzing disease- and group-describing parameters to explore factors that influence a disease's representation on Facebook.

These results may offer important insights for future public health initiatives and give direction to further research which can improve much needed support of parents of children with rare diseases. The analysis of Facebook groups dedicated to rare pediatric diseases and their development over time, for example, shows how many groups and individuals could benefit from an optimization of support groups conditions on Facebook. Initiatives that aim to promote communication among affected families can use this analysis to learn about support group structures such as group sizes and privacy settings. Having built the foundation of a quantitative analysis, future research can, for example, focus on a more in-depth qualitative analysis of Facebook group. Furthermore, this study points to the need for health professionals who treat individuals with rare pediatric disorders or affected parents or caregivers or provide genetic counseling to get better acquainted with the topic of social media support groups in order to understand and promote the communication among parents or caregivers of children with rare disorders. 


\section{Methods}

\section{Data Collection}

Rare diseases with childhood manifestation were identified using the inventory of the online portal for rare diseases and orphan drugs Orphanet). Orphanet uses the European definition of rare disease [22]. A rare pediatric disease is defined as a disease with onset before adulthood; thus, age of onset had to be defined as antenatal, conatal/neonatal, infancy, childhood or adolescent but not adult, older adult, or all ages. Data collected included disease name, 4 synonyms, ORPHAcode, Online Mendelian Inheritance in Man number, International Statistical Classification of Diseases Tenth Revision, disease prevalence, inheritance mode (autosomal or sex-linked, recessive or dominant, etc), age of onset and disease type (monogenic, deletion or alteration of a single gene; chromosomal, alteration in the number or structure of a chromosome; microdeletion, deletion of a small chromosomal segment; teratogenic disorder, result of exposure to teratogenic agent; mitochondrial; infectious disease; multigenic or multifactorial). Age of onset and disease type information was extracted from disease name or Orphanet disease description. Data were collected between January 1, 2019 and March 13, 2019.

Facebook support groups were identified by searching 5 synonymous disease names or descriptions using the Facebook group category search bar. The researcher used a Facebook account, newly created for this purpose, that contained only the researcher's name, picture, gender (female) and location (Cologne, Germany); but no activity (likes, shares, etc) expect for searching for aforementioned disease names. Groups were subcategorized according to their specific focus using the information available from the group title, the group category provided by Facebook's group categorization or the publicly available group description. Groups had to be clearly recognizable as support groups, groups to raise awareness and information, or support groups for individual patients. Groups that explicitly focused on research, fundraising and charity, medication sales, and disease-related pages were excluded from analysis (examples for categorization using group title Disorder $A$ fundraiser was categorized as focus on fundraising and excluded from analysis, Child B's journey with disorder $C$ was categorized as personal support group, Disorder D: spread awareness was categorized as awareness and information group). Data collected on Facebook included group type, name, language, privacy setting (public or private), foundation date, member count, and group insights. Group insights are provided by Facebook and displayed on each group information page, regardless of privacy status. They report activity (sum of posts, comments, and reactions calculated by Facebook) and new members during the past 30 days. Only groups with at least 1 member qualified. Foundation dates for groups on Facebook can be entered automatically or manually; manually entered foundation dates before Facebook launch in 2004 were excluded from analysis due to a lack of reliability of information. Data were collected between March 13, 2019 and March 31, 2019.

\section{Data Analysis}

Data were analyzed using standard descriptive statistical methods using SPSS statistics (version 26; IBM Corp). Normally distributed data are presented using mean and standard deviation, skewed distributions are presented using median and interquartile range, binary and categorical variables are presented using counts and percentages. Odds ratios (ORs) were calculated. The 1-sample Kolmogorov-Smirnov test was applied to test for normal distribution. Nonparametric tests (Spearman $\rho$ correlation, independent sample Mann-Whitney $U$ test) were applied. Binomial and chi-square tests were used to investigate binary and categorical variables.

This study has been reviewed by the Ethics Commission of the Medical Faculty of the University of Cologne (protocol 19-1027), and all research has been carried out within the scope of the approval.

\section{Results}

\section{General}

A total of 4246 rare disorders with onset before adulthood were identified using the Orphanet inventory, and 6398 support groups were found on Facebook. These groups represent 826 diseases, which amounts to $19.5 \%$ of all searched diseases.

The 10 diseases for which the most Facebook support groups were found are Down syndrome (145/6398, 2.3\%), 22q11.2 deletion syndrome $(117 / 6398,1.8 \%)$, hypoplastic left heart syndrome $(117 / 6398,1.8 \%)$, Turner syndrome $(97 / 6398,1.5 \%)$, gastroschisis $(95 / 6398,1.5 \%)$, cleft lip and palate $(93 / 6398$, $1.5 \%)$ Ehlers-Danlos syndrome (93/6398, 1.5\%), craniosynostosis $(92 / 6398,1.4 \%)$, microtia $(90 / 6398,1.4 \%)$, and retinoblastoma $(89 / 6398,1.4 \%)$. An alphabetic list of diseases on Facebook, including Online Mendelian Inheritance in Man number and the respective number of groups, is presented in Multimedia Appendix 1.

The total number of Facebook groups has continuously increased since 2008 (Figure 1). The number of newly created Facebook support groups shows fluctuation with an overall increase (Multimedia Appendix 2). 
Figure 1. Development of the total number of Facebook support groups for pediatric rare diseases over time.

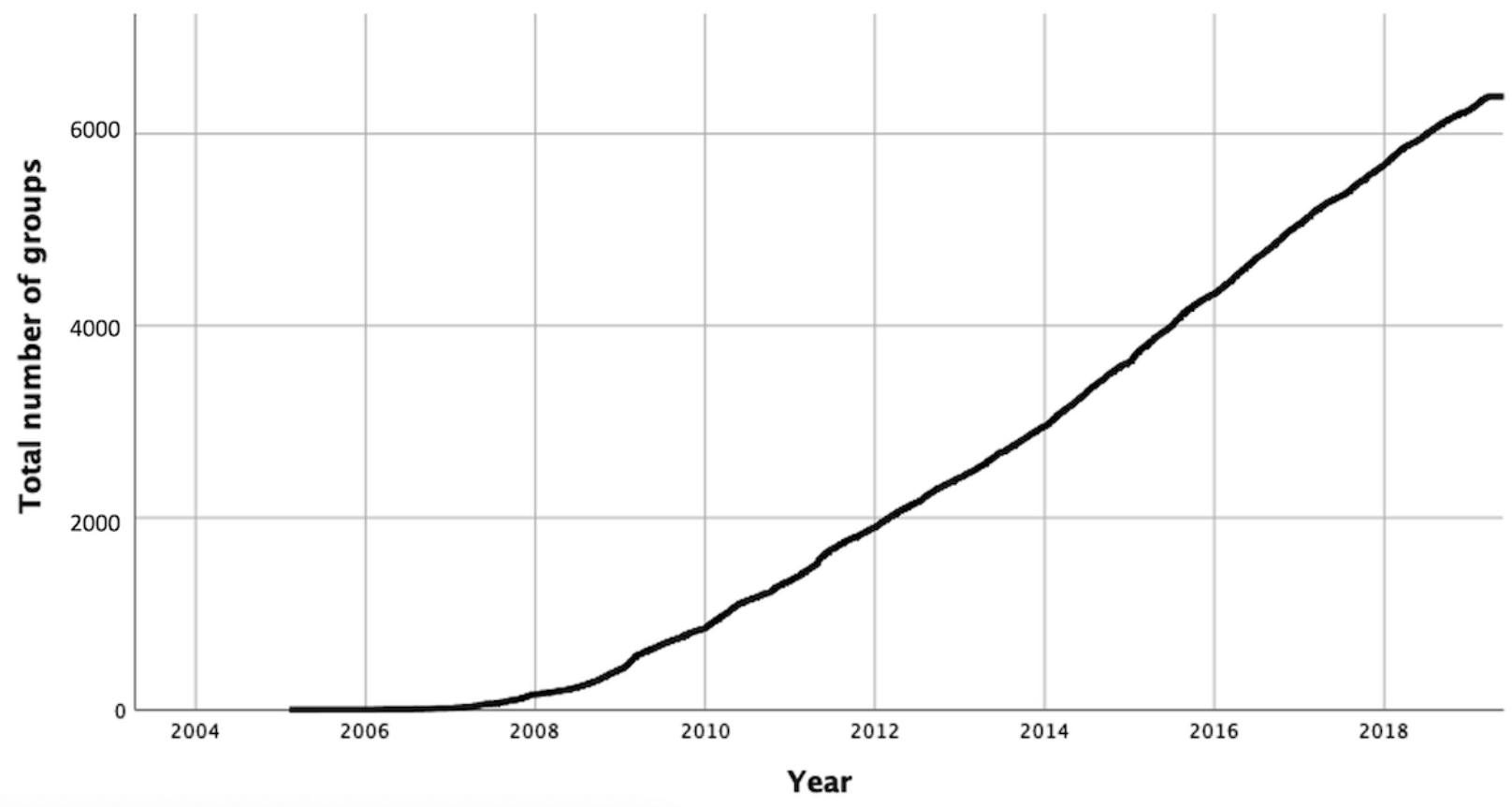

\section{Disease-Describing Parameters}

Of the 4246 diseases listed on Orphanet, 529 (12.9\%) diseases show antenatal and 2815 (68.9\%) show conatal/neonatal onset, $2167(53.0 \%)$ start during infancy, 1074 (26.3\%) during childhood, and $165(4.0 \%)$ show adolescent onset. More than one age of onset may apply.

Table 1 depicts the distribution of disease type and prevalence among all identified diseases and diseases with at least 1 support group and the number and percentage of Facebook support groups per disease type and prevalence. 274/934 (29.3\%) of the monogenic diseases, $51 / 145(35.2 \%)$ of the chromosomal, $85 / 158(53.8 \%)$ of the multigenic or multifactorial, and $4 / 22$ $(18.2 \%)$ of the teratogenic diseases found on Orphanet are represented on Facebook, and 376/2830 (13.3\%) of the diseases with unknown disease type. The mean number of groups per disease increases with increasing prevalence (mean 2 for prevalence <1 per 1,000,000; mean 5 for prevalence 1-9 per $1,000,000$; mean 14 for prevalence $1-9$ per 100,000 ; mean 28 for prevalence 1-9 per 10,000).

Table 2 displays the number and percentage of diseases on Orphanet and diseases with at least one group following the different inheritance modes. More than one inheritance mode may apply.

ORs were calculated to compare the probability of a disease with known or unknown disease-describing parameters to be represented by at least one Facebook group: known prevalence (OR 3.98, 95\% CI 3.39-4.66, $P<.001$ ), known disease type (OR $3.15,95 \%$ CI 2.70-3.68, $P<.001)$, and known inheritance mode (OR 2.06, 95\% CI 1.68-2.52, $P<.001$ ) are all associated with higher odds of finding a Facebook group; as is dominant compared to nondominant inheritance (OR 2.05, 95\% CI 1.74-3.42, $P<.001)$. 
Table 1. Diseases and Facebook support groups by prevalence and type of disease.

\begin{tabular}{llll}
\hline & $\begin{array}{l}\text { Diseases found on Orphanet } \\
(\mathrm{n}=4246), \mathrm{n}(\%)\end{array}$ & $\begin{array}{l}\text { Facebook support groups } \\
(\mathrm{n}=6398), \mathrm{n}(\%)\end{array}$ & $\begin{array}{l}\text { Diseases with } \geq 1 \text { group } \\
(\mathrm{n}=826), \mathrm{n}(\%)\end{array}$ \\
\hline Prevalence & $2516(59.3)$ & $620(9.7)$ & $253(30.6)$ \\
$\quad<1$ per 1,000,000 & $150(3.5)$ & $452(7.1)$ & $85(10.3)$ \\
$1-9$ per 1,000,000 & $188(4.4)$ & $1825(28.5)$ & $132(16.0)$ \\
$1-9$ per 100,000 & $77(1.8)$ & $1251(19.6)$ & $0(0.0)$ \\
$1-9$ per 10,000 & $1(<0.1)$ & $0(0.0)$ & $312(37.8)$ \\
$1-9$ per 1000 & $1314(30.9)$ & $2250(35.2)$ & $274(33.2)$ \\
Unknown & & $1623(25.4)$ & $51(6.2)$ \\
Disease type & $934(22.0)$ & $655(10.2)$ & $2(0.4)$ \\
Monogenic & $145(3.4)$ & $11(0.2)$ & $1(0.1)$ \\
Chromosomal & $25(0.6)$ & $1(<0.1)$ & $85(10.3)$ \\
Mitochondrial & $2(<0.1)$ & $988(15.4)$ & $4(0.5)$ \\
Infectious disease & $158(3.7)$ & $68(1.1)$ & $33(4.0)$ \\
Multigenic or multifactorial & $22(0.5)$ & $290(4.5)$ & $376(45.5)$ \\
Teratogenic disorder or infectious fetopathy & $130(3.0)$ & $2762(43.2)$ & \\
$\begin{array}{l}\text { Micro-/contiguous gene deletion/duplication/tripli- } \\
\text { cation }\end{array}$ & $2830(66.7)$ & &
\end{tabular}

Table 2. Pediatric rare diseases on Orphanet and diseases with at least one group that follow the different inheritance modes (more than one may apply).

\begin{tabular}{lll}
\hline Type $^{\mathrm{a}}$ & $\begin{array}{l}\text { Pediatric rare diseases on Orphanet that follow this } \\
\text { inheritance mode, } \mathrm{n}(\%)\end{array}$ & $\begin{array}{l}\text { Pediatric rare diseases with } \leq 1 \text { group that follow } \\
\text { this inheritance mode, } \mathrm{n}(\%)\end{array}$ \\
\hline Autosomal recessive & $1765(55.6)$ & $314(38.0)$ \\
Autosomal dominant & $971(30.6)$ & $75(33.8)$ \\
X-linked recessive & $306(9.6)$ & $25(3.0)$ \\
X-linked dominant & $70(2.2)$ & $31(3.8)$ \\
Multigenic or multifactorial & $74(2.3)$ & $1(0.1)$ \\
Mitochondrial & $15(0.5)$ & $0(0.0)$ \\
Y-linked & $1(<0.01)$ & $197(23.8)$ \\
Inheritance not applicable & $513(16.2)$ & \\
\hline
\end{tabular}

${ }^{\mathrm{a}}$ More than one may apply.

\section{Group-Describing Parameters}

\section{Group Type}

The support groups are further divided into the following subcategories: general support groups (4385/6398, 68.5\%), personal support groups $(828 / 6398,12.9 \%)$, support groups with focus on awareness and information $(450 / 6398,7.0 \%)$, not further specified groups $(649 / 6398,10.1 \%)$, groups for several diseases $(86 / 6398,1.3 \%)$. The following were not included: main focus on research $(n=147)$, fundraising and charity $(n=338)$, and medication sales $(n=4)$. The highest percentage of awareness and information groups was found for teratogenic diseases $(18 / 68,26 \%)$.

\section{Group Language}

Disease names or synonyms were entered into the Facebook search bar in English. Groups were mostly English speaking $(5721 / 6398,89.4 \%)$, with a smaller number listing French (227/6398, 3.5\%), Spanish (99/6398, 1.5\%), German, Dutch, Portuguese, Swedish, Turkish, Polish, or Danish as the group language. In total, 38 different group languages were found (Multimedia Appendix 3).

\section{Group Statistics}

Group-describing parameters were not normally distributed. The sum of group members in all groups amounted to 1,784,435. Membership in more than one group was possible. The median number of members was 33 (IQR 183, Q1 5, Q3 188; for comparison: mean 278.91, SD 989.46). This varied between 
personal support groups (mean 87.50, IQR 238.75), general support groups (mean 44, IQR 217), and awareness and information groups (mean 15, IQR 105.25). The maximum group member count was 23,414 in a group for pediatric multiple sclerosis, the minimum was 1 member in 496 groups. Of these, 326 groups were the only Facebook support group for the respective disease. Of these groups, 268 (82.2\%) were general support groups, 12 (3.7\%) were personal support groups, $11(3.4 \%)$ were awareness and information groups, $5(1.5 \%)$ were groups for several diseases, and 30 (9.2\%) were not further specified groups.

Throughout all groups, 84,966 new posts, comments and reactions were found (range 3606, minimum 0 , maximum 3606; mean 0, IQR 3, Q1 0, Q3 3). In total, 4021 groups showed no group activity. 35,119 persons joined the identified Facebook support groups (range 1357, minimum 0, maximum 1357; mean 0, IQR 1, Q1 0, Q3 1).

\section{Privacy Settings}

When set to private, content, such as posts and pictures, is only accessible to members whose membership must be approved by a group administrator. Group title, group description, and group statistics including member count, new members last 30 days and activity last 30 days are always publicly available. Of the identified groups, 69\% (4414/6398) are private, and $31 \%$
(1984/6398) are public. The sum of group members was $1,468,102$ in private and 316,333 in public groups, with a maximum of 23,414 members in a private and 17,000 members in a public group. The median member count was higher in private (mean 46) than in public groups (mean 14). The median activity and new members in the 30 days prior to analysis was 0 for both private and public groups, with a sum of 78,023 activities in private and 6943 activities in public groups and a sum of 29,566 new members in private and 5553 new members in public groups. Performing an independent-sample Mann-Whitney $U$ test showed that the distribution of the group-describing parameters differed slightly between the 2 privacy settings with higher member count, activities last 30 days, and new members last 30 days in private groups (member count: $U=5,296,374, z=13.44, P<.001$, effect size $r=0.17$; activities last 30 days: $U=5,602,193, z=20.65, P<.001$, effect size $r=0.29$; new members last 30 days: $U=5,104,178, z=13.04$, $P<.001$, effect size $r=0.16$ ).

\section{Correlation Analyses of Group- and Disease-Describing Parameter}

Correlation analyses showing relations between group- and disease-describing parameters are displayed in Table 3. The minimal age of onset correlates neither with the number of groups per disease nor with the group member count.

Table 3. Spearman correlations between disease- and group-describing parameters.

\begin{tabular}{lcc}
\hline Interpretation, variables & $\rho$ & $P$ value \\
\hline Significant strong positive correlation & 0.769 \\
$\quad$ Recent group activity and number of new members & 0.530 \\
Significant moderate positive correlation & 0.691 \\
$\quad$ Prevalence and number of groups per disease & 0.628 \\
$\quad$ Group member count and recent group activity & $<.001$ \\
$\quad$ Group member count and new group members & 0.101 \\
Significant weak positive correlation & 0.111 \\
$\quad$ Prevalence and group member count & $<.001$ \\
$\quad$ Time that a group exists and member count & $<.001$ \\
No significant correlation & 0.011 \\
$\quad$ Time that a group exists and recent group activity & -0.002 \\
$\quad$ Time that a group exists and number of new members & -0.021 \\
$\quad$ Disease's minimal age of onset and number of groups per disease & .001 \\
$\quad$ Disease's minimal age of onset and group member count & .001 \\
\hline
\end{tabular}

\section{Discussion}

\section{Principal Findings}

Facebook is widely used as a tool for support groups for individuals affected by rare pediatric diseases. This study has shown that, for approximately every fifth rare pediatric disease, one can find an existing Facebook support group. Group type, size, activity, new memberships, privacy settings, and language vary largely between groups.
Within the first years after the launch of Facebook in 2004, only a few Facebook support groups for rare pediatric diseases were created. Starting 2008 and onward, the total number of Facebook groups has been following almost linear growth. Consequently, we expect the number of groups and the number of diseases represented on Facebook to further increase in the coming years.

\section{Facebook Support Group Subtypes}

Support group subcategories allow different group focus and benefits. Analyses of some general support groups have shown that group members give and receive informational and 
emotional support [19], exchange knowledge and advice [18], and benefit from the ability to connect with others via Facebook [16].

Personal support groups (about every eighth identified group) are dedicated to one specific child with a certain disorder. Information about this child's health is shared and discussed. This group format has similarities with a blog but offers more personal two-way communication and thereby opportunities for emotional support. These groups' creator and members may especially benefit from having a place to speak openly about the disease and feelings as well as from receiving emotional support, which are 2 main benefit categories identified by White and Dorman [9]. A possible explanation for why these groups show the highest median member count could be a different target group. While other support groups are usually joined by parents and other immediate family members [12,16-18,20], these groups are probably also joined by family friends, who receive health updates and offer comfort, but who do not have a child with a similar condition. It could be of interest to evaluate the impact of this method of receiving social support in the context of rare diseases, since such groups do not depend on disease prevalence and finding others with the same condition.

Almost every tenth identified support group also focuses specifically on creating awareness and providing information. This is in agreement with previous studies' findings: families of patients with rare disease often become involved in raising public awareness [10], and social media can increase rare disease awareness [23]. The highest percentage of awareness and information groups was found for teratogenic diseases. Many teratogenic diseases are preventable disorders and parents, caregiver, or patients might therefore utilize Facebook groups to spread awareness to prevent future cases of the same disease.

\section{Insights Gained From Group Statistics}

\section{Facebook Group Accessibility}

Even though we used English search terms, we found support groups in 38 different languages, indicating that Facebook support groups are a worldwide development. This supports that Facebook is a fitting tool for support groups since it is a globally accessible platform [13]. Facebook offers the possibility to easily and inexpensively share information 24 hours a day and time-zone independent [10,21]. Parents of children with rare diseases are active internet users and use social media such as Facebook [12,15]. Together with the aspect of internationality, Facebook is therefore accessible as a tool for support groups for many, if not most, caregivers for a child with a rare disease.

\section{Group Members and Activity}

Group sizes vary greatly. Group member count can be influenced by multiple factors, as we have shown for disease prevalence. Other factors may include group promotion among affected families and by health professionals.

Correlation analyses showed that groups with more group members also had slightly more recent group activity and new members. Support group participants could therefore benefit from joining a larger Facebook group, since it offers more active discussions and more individuals to connect with. Group activity and new member count showed a strong positive correlation, which indicates that new members start new conversations.

Many groups did not show any group activity. This could be either coincidental or indicate that these groups are inactive. Even if groups are formally inactive, there might still be private conversations between group members using Facebook Messenger or other personal messaging services, which we were not able to evaluate.

\section{Looking for Others to Start a Support Group}

Our analysis showed that 326 persons created a group for a specific disease, but at the time of study no one had joined their group, which means that they were unsuccessfully looking for someone to start a support group with. Possible reasons are that no one directly or indirectly affected by the same condition has turned to Facebook in order to look for a support group, or that no one is available for a support group on Facebook. Regarding the first explanation, it could be helpful to raise awareness of Facebook as a tool for rare pediatric disease support groups among parents and caregivers. A survey of caregivers of children with Autism Spectrum Disorders found that caregivers whose diagnosing clinician had referred them to a support group were more likely support group participants [20]. Therefore, health professionals ought to get better acquainted with the topic of social media support groups if they aim to promote the communication among parents and caregivers of children with rare disorders.

\section{Facebook Support Group Privacy}

A group's privacy setting limits access to the group. When set to private, membership must be validated by a group administrator before content such as posts and pictures can be accessed or created. Group description and group statistics are publicly available. Our analysis showed that two thirds of the groups were private, which is in agreement with a survey conducted among patients with newly described or rare genetic findings, of whom $60 \%$ were uncomfortable with sharing information in a public group [13].

Because of the need of a validation before joining a private group, we expected public groups to have higher member counts, but private groups' median member count resulted to be three times higher. More individuals appear to prefer joining private support groups, which indicates members' preference of a more private environment when sharing experiences related to their children's health.

Nevertheless, sharing information in a private Facebook group still means sharing information about a child online and oftentimes with (relative) strangers. Confidentiality and privacy issues are an important topic, since group participants are often unaware of risks of disclosing personal information [11]. Studies on mothers' habits of sharing private details on their children on Facebook regardless their children's health status have shown that mothers become increasingly aware of privacy issues on Facebook and try to find a balance between the need for privacy and the benefits of openness; some felt that some information was not appropriate to share $[24,25]$. This topic is particularly challenging since children cannot object to sharing information 
and pictures online, but might experience negative consequences later in life. Privacy issues therefore need to be investigated in the context of Facebook as a tool for rare pediatric disease support groups.

\section{Factors That Influence a Disease's Representation on Facebook}

The more individuals are affected by a disease, the more individuals potentially turn to Facebook to look for or create a support group. Many diseases with higher prevalence have several support groups, and these groups' descriptions often include geographic locations, eg different countries or states. Facebook group members have been shown to organize meetings for particular events [19], and Facebook groups organized according to members' locations could facilitate this.

The analysis of variables collected in this research showed that the odds of finding a Facebook group for a disease with known prevalence are almost four times higher compared to with unknown prevalence, for a disease with known inheritance two times higher than with unknown inheritance, and for a disease with known disease type more than three times higher than with unknown disease type. These findings suggest that the chances of finding a Facebook group increase with a higher level of understanding about the disease. New diseases are described regularly, but it takes time and resources to investigate newly described diseases, and for many rare diseases there is a subsequent deficit of medical and scientific knowledge [2]. It also takes time until more affected children have been diagnosed. This limited information and the factor of time influence the chance of finding a group on Facebook. Other factors may play a role as well. The impact of disease-specific mortality, for example, could be of great importance and might therefore be of interest for further investigation.

The odds of finding a Facebook support group for a disease with possible dominant inheritance are twice as high as for a disease without dominant inheritance. The risk of transmission in dominant diseases is $50 \%$. Individuals affected by dominant diseases therefore encounter several challenges, such as reproductive decision making, feelings of guilt, and the need to communicate genetic risk with their children and family members [26]. These challenges can influence an individual's need for peer support, which may, in part, explain the higher probability of finding groups for dominant diseases.

\section{Consequences for Treating Physicians and Other Health Care Professionals}

This study indicates a need for health care professionals to become acquainted with social media as a tool for support groups, since it is already widely used. To allow informed decision making on whether to refer parents and caregivers of children with a rare disease to Facebook, more research about the strengths and limitations of Facebook as a tool for support groups is needed. If treating physicians decide to promote Facebook support groups, they can inform patients about the chances of finding a group, which is 1 in 5 overall but higher for monogenic, chromosomal, and multigenic or multifactorial diseases. Physicians with a focus on certain diseases can use our research to explore the extent of Facebook usage for groups for the respective disease. Furthermore, this study strengthens the importance of further research about rare diseases, since knowledge about a rare disease also influences the availability of support groups.

\section{Study Strengths and Limitations}

This study is a broad-scope analysis. All Orphanet-listed diseases which conform to the inclusion criteria were searched on Facebook using 5 synonymous disease descriptions. Nevertheless, it is impossible to know whether all existing support groups have been identified. Even though the Facebook search was conducted in English, groups in 38 different languages were identified. It is possible that not all existing non-English groups have been found, especially when disease names differ largely from the English disease name. Other groups regardless of group language may not at all use a disease description in their group title. Facebook search engine optimization may have influenced our search results using the researcher's information regarding location and gender. To minimize this effect, no other activities such as viewing, liking or sharing have been performed. Group activity and the number of new memberships could vary and since this was a cross-sectional study our data may not be representative. This could be investigated by repeating the study at another point in time. The limited information on Orphanet also limited our analysis. Disease type was evident for only a third of the diseases and information on prevalence was provided for only two-thirds of the diseases.

\section{Conclusion}

There has been a continuous rise in the number of support groups and diseases represented on Facebook since 2008. We expect that the relevance of Facebook as a tool for rare pediatric disease support groups will continue to increase. Group type, size, activity, new memberships, privacy settings, and language vary largely between groups. Support group subcategories allow different group focus. The odds of finding a Facebook group have been shown to increase according to the level of information available about the disease, and the number of groups increases with higher prevalence. Two-thirds of the groups are private groups, indicating the group participants' need for privacy, which should be further explored. More research is necessary to investigate the strengths and limitations of Facebook as a tool for support groups in the field of rare disease. This will allow health professionals to use Facebook more meaningfully in their counseling and guidance of affected individuals and their family members. It may also allow Facebook and other similar social media platforms to improve their toolkits and offerings for individuals affected by rare diseases. 


\section{Acknowledgments}

The authors thank Rebecca Roth, Dr. Sc. Hum., Institute of Medical Statistics and Bioinformatics, Medical Faculty, University of Cologne, for the statistical consultancy support. Andrea Plaza-Gonzalez and Karin Mombaur are acknowledged for proofreading and editing the manuscript.

\section{Conflicts of Interest}

None declared.

\section{Multimedia Appendix 1}

Alphabetic list of diseases.

[DOC File, 711 KB-Multimedia Appendix 1]

\section{Multimedia Appendix 2}

The number of newly created Facebook support groups shows fluctuation with an overall increase.

[PNG File , 60 KB-Multimedia Appendix 2]

\section{Multimedia Appendix 3}

Full list of Facebook support groups by language.

[DOC File, $51 \mathrm{~KB}-$ Multimedia Appendix 3]

\section{References}

1. Pelentsov LJ, Fielder AL, Esterman AJ. The supportive care needs of parents with a child with a rare disease: a qualitative descriptive study. J Pediatr Nurs 2016;31(3):e207-e218. [doi: 10.1016/j.pedn.2015.10.022] [Medline: 26651231]

2. About rare diseases. Orphanet. URL: https://www.orpha.net/consor/cgi-bin/Education AboutRareDiseases.php?lng=EN [accessed 2019-12-20]

3. Shilling V, Morris C, Thompson-Coon J, Ukoumunne O, Rogers M, Logan S. Peer support for parents of children with chronic disabling conditions: a systematic review of quantitative and qualitative studies. Dev Med Child Neurol 2013 Jul;55(7):602-609 [FREE Full text] [doi: 10.1111/dmcn.12091] [Medline: 23421818]

4. Blake L, Bray L, Carter B. Patient Educ Couns 2019 Dec;102(12):2279-2285 [FREE Full text] [doi: 10.1016/j.pec.2019.07.012] [Medline: 31327482 ]

5. Solomon M, Pistrang N, Barker C. The benefits of mutual support groups for parents of children with disabilities. Am J Community Psychol 2001 Feb;29(1):113-132. [doi: 10.1023/A:1005253514140] [Medline: 11439824]

6. McConnell D, Savage A, Breitkreuz R. Resilience in families raising children with disabilities and behavior problems. Res Dev Disabil 2014 Apr;35(4):833-848. [doi: 10.1016/j.ridd.2014.01.015] [Medline: 24491480]

7. Cantwell J, Muldoon OT, Gallagher S. Social support and mastery influence the association between stress and poor physical health in parents caring for children with developmental disabilities. Res Dev Disabil 2014 Sep;35(9):2215-2223. [doi: 10.1016/j.ridd.2014.05.012] [Medline: 24927515]

8. Giustini D, Ali SM, Fraser M, Kamel Boulos MN. Effective uses of social media in public health and medicine: a systematic review of systematic reviews. Online J Public Health Inform 2018;10(2):e215 [FREE Full text] [doi: 10.5210/ojphi.v10i2.8270] [Medline: $\underline{30349633}$ ]

9. White M, Dorman SM. Receiving social support online: implications for health education. Health Educ Res 2001 Dec;16(6):693-707. [doi: 10.1093/her/16.6.693] [Medline: 11780708 ]

10. Delisle VC, Gumuchian ST, Rice DB, Levis AW, Kloda LA, Körner A, et al. Perceived benefits and factors that influence the ability to establish and maintain patient support groups in rare diseases: a scoping review. Patient 2017 Jun 21;10(3):283-293. [doi: 10.1007/s40271-016-0213-9] [Medline: 28004275]

11. Moorhead SA, Hazlett DE, Harrison L, Carroll JK, Irwin A, Hoving C. A new dimension of health care: systematic review of the uses, benefits, and limitations of social media for health communication. J Med Internet Res 2013 Apr 23;15(4):e85 [FREE Full text] [doi: 10.2196/jmir.1933] [Medline: 23615206]

12. Nicholl H, Tracey C, Begley T, King C, Lynch AM. Internet use by parents of children with rare conditions: findings from a study on parents' web information needs. J Med Internet Res 2017 Feb 28;19(2):e51 [FREE Full text] [doi: 10.2196/jmir.5834] [Medline: 28246072]

13. Rocha HM, Savatt JM, Riggs ER, Wagner JK, Faucett WA, Martin CL. Incorporating social media into your support tool box: points to consider from genetics-based communities. J Genet Couns 2018 Apr 13;27(2):470-480. [doi: 10.1007/s10897-017-0170-z] [Medline: 29130143]

14. Facebook Reports Second Quarter 2019 Results. Facebook Inc. 2019. URL: https://s21.q4cdn.com/399680738/files/ doc financials/2019/Q2/FB-Q2-2019-Earnings-Release.pdf [accessed 2019-12-20] 
15. Tozzi AE, Mingarelli R, Agricola E, Gonfiantini M, Pandolfi E, Carloni E, et al. The internet user profile of Italian families of patients with rare diseases: a web survey. Orphanet J Rare Dis 2013 May 16;8:76 [FREE Full text] [doi: 10.1186/1750-1172-8-76] [Medline: 23680013]

16. Stock NM, Martindale A, Cunniffe C, VTCT Foundation Research Team at the Centre for Appearance Research. \#CleftProud: a content analysis and online survey of 2 cleft lip and palate Facebook groups. Cleft Palate Craniofac J 2018 Nov;55(10):1339-1349. [doi: 10.1177/1055665618764737] [Medline: 29570382]

17. Oprescu F, Campo S, Lowe J, Andsager J, Morcuende JA. Online information exchanges for parents of children with a rare health condition: key findings from an online support community. J Med Internet Res 2013 Jan;15(1):e16 [FREE Full text] [doi: 10.2196/jmir.2423] [Medline: 23470259]

18. Wittmeier K, Holland C, Hobbs-Murison K, Crawford E, Beauchamp C, Milne B, et al. Analysis of a parent-initiated social media campaign for Hirschsprung's disease. J Med Internet Res 2014 Dec 11;16(12):e288 [FREE Full text] [doi:

10.2196/jmir.3200] [Medline: 25499427]

19. Mohd Roffeei SH, Abdullah N, Basar SKR. Seeking social support on Facebook for children with Autism Spectrum Disorders (ASDs). Int J Med Inform 2015 May;84(5):375-385. [doi: 10.1016/j.ijmedinf.2015.01.015] [Medline: 25701266]

20. Mandell DS, Salzer MS. Who joins support groups among parents of children with autism? Autism 2007 Mar;11(2):111-122 [FREE Full text] [doi: 10.1177/1362361307077506] [Medline: 17353212]

21. Black A, Baker M. The impact of parent advocacy groups, the Internet, and social networking on rare diseases: the IDEA League and IDEA League United Kingdom example. Epilepsia 2011 Apr;52 Suppl 2:102-104 [FREE Full text] [doi: 10.1111/j.1528-1167.2011.03013.x] [Medline: 21463291]

22. Search for a rare disease. Orphanet. 2019. URL: https://www.orpha.net/consor4.01/www/cgi-bin/Disease.php?lng=EN [accessed 2019-12-05]

23. Milne C, Ni W. The use of social media in orphan drug development. Clin Ther 2017 Nov;39(11):2173-2180. [doi: 10.1016/j.clinthera.2017.08.016] [Medline: 28942336]

24. Holtz B, Smock A, Reyes-Gastelum D. Connected motherhood: social support for moms and moms-to-be on Facebook. Telemed J E Health 2015 May;21(5):415-421. [doi: 10.1089/tmj.2014.0118] [Medline: 25665177]

25. Chalklen C, Anderson H. Mothering on Facebook: exploring the privacy/openness paradox. Social Media + Society 2017 May 17;3(2):205630511770718. [doi: 10.1177/2056305117707187]

26. Metcalfe A, Plumridge G, Coad J, Shanks A, Gill P. Parents' and children's communication about genetic risk: a qualitative study, learning from families' experiences. Eur J Hum Genet 2011 Jun;19(6):640-646 [FREE Full text] [doi:

10.1038/ejhg.2010.258] [Medline: 21326287]

Edited by G Eysenbach; submitted 22.06.20; peer-reviewed by P Magoulas, W Davies; comments to author 11.07.20; revised version
received 21.07.20; accepted 28.10.20; published 19.11.20
Please cite as:
Titgemeyer SC, Schaaf CP
Facebook Support Groups for Rare Pediatric Diseases: Quantitative Analysis
JMIR Pediatr Parent 2020;3(2):e21694
URL: $\underline{\text { http://pediatrics.jmir.org/2020/2/e21694/ }}$
doi: $\underline{10.2196 / 21694}$
PMID: $\underline{3211019}$

(CSarah Catrin Titgemeyer, Christian Patrick Schaaf. Originally published in JMIR Pediatrics and Parenting (http://pediatrics.jmir.org), 19.11.2020. This is an open-access article distributed under the terms of the Creative Commons Attribution License (https://creativecommons.org/licenses/by/4.0/), which permits unrestricted use, distribution, and reproduction in any medium, provided the original work, first published in JMIR Pediatrics and Parenting, is properly cited. The complete bibliographic information, a link to the original publication on http://pediatrics.jmir.org, as well as this copyright and license information must be included. 